\title{
Genome organization is not conserved between Bacillus cereus and Bacillus subtilis
}

\author{
Ole A. Økstad, Ida Hegna, Toril Lindbäck, Anne-Lise Rishovd \\ and Anne-Brit Kolstø
}

Author for correspondence: Anne-Brit Kolstø. Tel: +4722958460. Fax: +4722694130.
e-mail: annebko@ biotek.uio.no

Biotechnology Centre of Oslo and School of Pharmacy, University of Oslo, $\mathrm{Pb} 1125$ Blindern, 0316 Oslo, Norway

\begin{abstract}
The opportunistic pathogen Bacillus cereus is the genetically stable member of a group of closely related bacteria including the insect pathogen Bacillus thuringiensis and the mammalian pathogen Bacillus anthracis. Physical maps of $B$. cereus and B. thuringiensis strains show considerable variations in discrete parts of the chromosome, suggesting that certain genome regions are more prone to rearrangements. $B$. cereus belongs to the same subgroup of Bacillus species as Bacillus subtilis, by both phenotypic and rRNA sequence classification. The analysis of $\mathbf{8 0} \mathbf{~ k b}$ of genome sequence sampled from different regions of the B. cereus ATCC 10987 chromosome is reported. Analysis of the sequence and comparison of the localization of the putative genes with that of $B$. subtilis orthologues show the following: (1) gene organization is not conserved between B. cereus and B. subtilis; (2) several putative genes are more closely related to genes from other bacteria and archaea than to $B$. subtilis, or may be absent in $B$. subtilis 168 ; (3) B. cereus contains a 155 bp repetitive sequence that is not present in $B$. subtilis. By hybridization, this repeat is present in all B. cereus and B. thuringiensis strains so far investigated.
\end{abstract}

Keywords: comparative genome analysis, gene organization, repeated sequence, Bacillus cereus, Bacillus subtilis

\section{INTRODUCTION}

Several studies in comparative genomics using whole genomes have been performed. These have focused mainly on distantly related bacteria (Koonin et al., 1996; Tatusov et al., 1996; Kolstø, 1997; Smith et al., 1997). In addition, the close relatives Mycoplasma genitalium and Mycoplasma pneumoniae have been analysed (Himmelreich et al., 1997). By sequence comparison, the Mycoplasma genomes could be subdivided into six DNA segments which were ordered differently in the two species, suggested to have originated by translocation via homologous recombination. The order of the genes within each segment was conserved, and the increased size of the M. pneumoniae chromosome appeared to be due mainly to gene duplications (Himmelreich et al., 1997).

Bacillus cereus is a Gram-positive, rod-shaped, endospore-forming bacterium commonly found in soil, air

The EMBL accession numbers for the sequences reported in this paper are specified in Table 1. and water, and is a frequent cause of food-related diseases (Drobniewski, 1993). It is also an opportunistic pathogen involved in local and systemic infections. The pathogenicity of $B$. cereus is caused by its synthesis of several virulence factors, including a putative collagenase, phospholipases, haemolysins, diarrhoeagenic enterotoxins and emetic toxin (Drobniewski, 1993). Bacillus thuringiensis is distinguished from $B$. cereus by its production of insecticidal crystal toxins (reviewed by Aronson, 1993). Both species belong to the $B$. cereus group, which also includes the closely related Bacillus anthracis and Bacillus mycoides. Accumulated data have suggested that $B$. cereus should be considered the parent species of the group, and that the other species should be regarded as subspecies (Smith et al., 1952; Gordon et al., 1973). All members of the $B$. cereus group have been allocated to the B. subtilis group of bacilli, by both phenotypic and rRNA classification (Priest, 1993). More than 19 species were assigned to the group, for which B. subtilis was chosen as an appropriate representative.

Previously we have constructed physical maps of eight 
B. cereus and four B. thuringiensis strains using pulsedfield gel electrophoresis (PFGE) and primarily anonymous fragments of HindIII-digested chromosomal DNA as hybridization probes (Kolstø et al., 1990; Carlson et al., 1992, 1996a, b; Carlson \& Kolstø, 1993, 1994; Lövgren et al., 1998; K. Kvaløy \& A.-B. Kolstø, unpublished results). The size of the chromosome was found to display a $2 \cdot 6$-fold size variation, and it was suggested that $B$. cereus and B. thuringiensis chromosomes contain one region in which the gene order is more conserved whereas other regions exhibit a more variable gene organization. Sequencing of the anonymous probes used for physical mapping has now enabled us to analyse and map 64 chromosomal genes from $B$. cereus ATCC 10987, and to compare the gene organization to its relative, Bacillus subtilis 168 , for which the complete genome sequence is known (Kunst et al., 1997). Contradictory to the two complete Mycoplasma genomes, the organization of orthologues in B. cereus and B. subtilis was not conserved, and no common arrangement pattern could be seen.

\section{METHODS}

Bacterial strain. B. cereus ATCC 10987 was obtained from the American Type Culture Collection, Manassas, VA, USA. The strain is a xylose-positive variant previously used in our laboratory due to its high level of phospholipase $\mathrm{C}$ production.

DNA sequencing and analysis. Plasmid DNA from previously prepared randomly selected clones of HindIII-digested genomic DNA from B. cereus ATCC 10987 (Kolstø et al., 1990) was isolated using standard methods (Sambrook et al., 1989) and sequenced by the dideoxynucleotide method (Sanger $e t$ al., 1977) using the ThermoSequenase Kit (Amersham) and fluorescein end-labelled oligonucleotide primers on the ALF automated sequencer (Pharmacia). Oligonucleotide primers were prepared at the DNA Synthesis Laboratory, Biotechnology Centre of Oslo. DNA sequences were analysed using the GCG and EGCG sequence analysis programs (Devereux et al., 1984). Sequences were edited using SEQED, ORFs and restriction sites were detected using MAP and translated using TRANSLATE. Translated ORFs were used to search the SWISSPROT (release 34.0,11/96), TREMBL (release 3.0, 05/97) and SubtiList (Data Release R14.2, 11/20/97) protein databases by FASTA (Pearson \& Lipman, 1988), and by BLAST and GAPPED BLAST (Altschul et al., 1990, 1997) to search the nonredundant database set of protein entries at NCBI, Bethesda, $\mathrm{MD}$, USA. COMposition was used to determine the $\mathrm{G}+\mathrm{C}$ content of the DNA sequences, and multiple sequence alignments were constructed by PILEUP (Needleman \& Wunsch, 1970; Feng \& Doolittle, 1987; Higgins \& Sharp, 1989).

PFGE, Southern blotting and DNA hybridization. Intact chromosomal DNA was prepared in agarose blocks and PFGE was run using the Bio-Rad CHEF Gene Mapper (Bio-Rad) or the Beckman GeneLine I apparatus (Beckman Instruments), as described by Carlson et al. (1992). Southern blotting and DNA hybridization using gene probes was performed as previously described (Carlson et al., 1992). The oligonucleotide probe for bcr1 $\left(5^{\prime}\right.$ CTC AAA ATT CAG CGG AAG CAA AGA ANT TAG GTG GGG G 3', where $\mathrm{N}$ is $\mathrm{A}, \mathrm{G}, \mathrm{C}$ or $\mathrm{T}$ ) was prepared at the DNA Synthesis Laboratory, Biotechnology Centre of Oslo. For hybridization, $100 \mathrm{ng}$ of the probe $(1 \mu \mathrm{l})$ was endlabelled using $2 \mu \mathrm{l}\left[\gamma_{-}{ }^{32} \mathrm{P}\right] \mathrm{dATP}\left[5000 \mathrm{Ci} \quad\left(18.5 \times 10^{13} \mathrm{~Bq}\right)\right.$ $\mathrm{mmol}^{-1}$; Amersham] and $10 \mathrm{U} \mathrm{T} 4$ polynucleotide kinase (New England Biolabs) in $1 \times \mathrm{T} 4$ polynucleotide kinase reaction buffer and $10 \mu \mathrm{l}$ reaction volume at $37^{\circ} \mathrm{C}$ for $1 \mathrm{~h}$. Membrane filters were pre-hybridized in $6 \times \mathrm{SSC}, 10 \times$ Denhardt's solution, $0.2 \%$ SDS at $50{ }^{\circ} \mathrm{C}$ for $30 \mathrm{~min}$, and hybridized for $1.5 \mathrm{~h}$ at room temperature following addition of the end-labelled probe. Filters were washed at room temperature for $30 \mathrm{~min}$, followed by $15 \mathrm{~min}$ at $50^{\circ} \mathrm{C}$ and $15 \mathrm{~min}$ at $62^{\circ} \mathrm{C}$, and exposed and scanned using a Storm 860 Phosphorimager (Molecular Dynamics).

\section{RESULTS AND DISCUSSION}

We have partially or completely sequenced 43 of the anonymous probes used for the construction of physical maps of $B$. cereus and $B$. thuringiensis. The probes covered a total sequence length of $80 \mathrm{~kb}$, and 86 open reading frames (ORFs) were identified (Table 1). Seventy-eight ORFs showed similarity ( $>20 \%$ amino acid identity) to submitted entries in the protein sequence databases, and 54 were assigned a putative function by similarity to proteins of known or predicted function. Three ORFs were assigned functions in DNA repair, after complementing the DNA alkylation repair deficient Escherichia coli strain BK2118 (alkC, alkD and alkE; I. Alseth and others, unpublished results). Ten ORFs matched proteins of unknown function in the databases, eight of which were from B. subtilis. Fourteen ORFs displayed weak sequence similarity, whereas eight ORFs showed no similarity to any database entries. The G+C content was calculated to $35.4 \mathrm{~mol} \%$, in agreement with previous predictions from DNA reassociation experiments (Priest, 1993).

\section{Isolated genes novel to bacilli}

Seven of the 54 putative genes of predicted function in $B$. cereus ATCC 10987 encoded proteins with no sequence homologues in other Bacillus spp. (Table 1). These included a type III restriction-modification system $(t 3 r$; Hegna et al., 1992) resembling systems from the Gramnegative bacteria Salmonella typhimurium and E. coli phage P1, and one of the DNA alkylation repair enzymes (alkD; I. Alseth and others, unpublished results). Moreover, the protein encoded by cys $A$ resembled sulfate transporters from cyanobacteria (Synechococcus PCC 7942) and eubacteria (E. coli) (Green et al., 1989), but showed no similarity to $B$. subtilis proteins. No protein participating in sulfate transport has to our knowledge been identified in $B$. subtilis, although several sulfate metabolism enzymes are found (SubtiList, Data Release R14.2, 1998; www server v2.1.3: http://www. pasteur.fr/Bio/SubtiList.html). The B. cereus glyS gene encoded a homologue of class II aminoacyl-tRNA synthetases (Logan et al., 1995). B. subtilis and B. cereus GlyS were of separate class II subtypes that are not sequence related (SubtiList, Data Release R14.2, 1998).

Recently, a number of bacteria have been identified that are capable of metabolizing atrazine, a triazine ringcontaining herbicide that has been found to be less 
Table 1. Analysis of $80 \mathrm{~kb}$ DNA sequence from B. cereus ATCC 10987

\begin{tabular}{|c|c|c|c|c|c|c|c|}
\hline $\begin{array}{l}\text { Accession } \\
\text { no. }\end{array}$ & Fragment & Length (bp) & $\begin{array}{c}\text { Gene } \\
\text { homologues }\end{array}$ & $\begin{array}{l}\text { Putative } \\
\text { function* }\end{array}$ & $\begin{array}{c}\text { Identity } \\
(\%) \dagger\end{array}$ & $\begin{array}{l}\text { Amino } \\
\text { acid } \\
\text { overlap }\end{array}$ & $\begin{array}{c}\text { Organism/Accession } \\
\text { no. }\end{array}$ \\
\hline Y11217 & agt85 & 763 & add $A$ & $\begin{array}{l}\text { ATP-dependent } \\
\text { nuclease subunit A }\end{array}$ & 50 & $253 / 255$ & B. subtilis $\mathrm{Sw}$ : P23478 \\
\hline Y09433 & bc522 & 1373 & $r s 1 b$ & $\begin{array}{l}\text { 30S ribosomal } \\
\text { protein } \mathrm{S} 1\end{array}$ & 63 & $381 / 381$ & $\begin{array}{l}\text { B. subtilis } \mathrm{Sw} \text { : } \\
\text { P38494 }\end{array}$ \\
\hline Y09253 & bc67 & 1099 & $y f b M$ & $\begin{array}{l}\mathrm{U} \text { (similarity to } \\
\text { alkaline- } \\
\text { phosphatase-like } \\
\text { protein) }\end{array}$ & 36 & $158 / 203$ & B. subtilis $\mathrm{Gb}$ : Z99105 \\
\hline Y09254 & bc 87 & 367 & $b c 87 a$ & $\mathrm{U}$ & & & \\
\hline \multirow[t]{3}{*}{ Y11139 } & bc204 & 2994 & $b c 204 a$ & $\mathrm{U}$ & & & \\
\hline & & & $b c 204 b$ & $\mathrm{U}$ & & & \\
\hline & & & yor 3 & $\begin{array}{l}\text { Putative } N \text { - } \\
\text { ethylammeline } \\
\text { chlorohydrolase }\end{array}$ & 44 & $284 / 312$ & $\begin{array}{l}\text { Methanobacterium } \\
\text { thermoautotrophicum } \\
\text { Gb: AE000910 }\end{array}$ \\
\hline Y08953 & bc 41 & 829 & $\mathrm{cmk}$ & Cytidylate kinase & 70 & $193 / 199$ & B. subtilis $\mathrm{S}_{\mathrm{w}}: \mathrm{P} 38493$ \\
\hline Y11141 & bc532 & 431 & colA & $\begin{array}{l}\text { Putative collagenase } \\
\text { precursor }\end{array}$ & 36 & $78 / 143$ & $\begin{array}{l}\text { C. perfringens Sw: } \\
\text { P43153 }\end{array}$ \\
\hline \multirow[t]{3}{*}{ Y09212 } & bc72 & 3478 & glt $T$ & $\begin{array}{l}\text { Proton/sodium- } \\
\text { glutamate symport } \\
\text { protein }\end{array}$ & 59 & $355 / 355$ & B. subtilis $\mathrm{Sw}$ : $\mathrm{P} 24943$ \\
\hline & & & $a n s B$ & $\begin{array}{l}\text { Aspartate ammonia- } \\
\text { lyase }\end{array}$ & 79 & $467 / 478$ & B. subtilis $\mathrm{Sw}_{\mathrm{w}}$ : 226899 \\
\hline & & & mals & $\begin{array}{l}\text { Malate } \\
\text { dehydrogenase }\end{array}$ & 71 & $291 / 291$ & B. subtilis Em: Z99119 \\
\hline Y09252 & bc 92 & 1131 & purH & $\begin{array}{l}\text { Phosphoribosylamino- } \\
\text { imidazolecarboxamide- } \\
\text { formyltransferase }\end{array}$ & 74 & $335 / 337$ & B. subtilis $\mathrm{Sw}$ : P12048 \\
\hline \multirow[t]{3}{*}{ Y11138 } & bc205 & 2402 & $b c 205 a$ & $\begin{array}{l}\mathrm{U} \text { (similarity to } \\
\text { capsule cluster gene } \\
\text { cap5J) }\end{array}$ & 19 & $358 / 405$ & S. aureus Gb: U81973 \\
\hline & & & $b c 205 b$ & $\mathrm{U}$ & & & \\
\hline & & & $b c 205 c$ & $\begin{array}{l}\mathrm{U} \text { (similarity to } \\
\text { galactosamine- } \\
\text { containing minor } \\
\text { teichoic acid } \\
\text { biosynthesis protein } \\
\text { GgaB) }\end{array}$ & 64 & $14 / 21$ & B. subtilis Sw: P46918 \\
\hline \multirow[t]{2}{*}{ Y10981 } & bc45 & 865 & proA & $\begin{array}{l}\gamma \text {-Glutamyl } \\
\text { phosphate reductase }\end{array}$ & 67 & $247 / 247$ & B. subtilis $\mathrm{Sw}$ : P39821 \\
\hline & & & $y x j A$ & $\begin{array}{l}\text { Putative pyrimidine } \\
\text { nucleoside transport } \\
\text { protein }\end{array}$ & 86 & $22 / 22$ & B. subtilis $\mathrm{S}_{\mathrm{w}}$ : $\mathrm{P} 42312$ \\
\hline \multirow[t]{2}{*}{ Y11170 } & bc541 & 842 & $y t d I$ & $\mathrm{U}($ similarity to $y t d I)$ & 58 & $177 / 184$ & $\begin{array}{l}\text { B. subtilis Gb: } \\
\text { AF008220 }\end{array}$ \\
\hline & & & $y w j E$ & $\begin{array}{l}\mathrm{U} \text { (similarity to } \\
y w j E)\end{array}$ & 50 & $20 / 24$ & B. subtilis $\mathrm{S}_{\mathrm{w}}$ : $\mathrm{P} 45865$ \\
\hline Y10909 & bc73 & 1368 & $s p 5 k \_1$ & $\begin{array}{l}\text { Similarity to stage } \mathrm{V} \\
\text { sporulation protein } \\
\mathrm{K}\end{array}$ & 40 & $275 / 455$ & B. subtilis $\mathrm{Sw}: \mathrm{P} 27643$ \\
\hline Y1130.5 & glpT & 485 & $g l p T$ & $\begin{array}{l}\text { Glycerol-3-phosphate } \\
\text { permease }\end{array}$ & 71 & $153 / 161$ & $\begin{array}{l}\text { B. subtilis Sw: } \\
\text { P37948 }\end{array}$ \\
\hline Y09322 & bc 331 & 802 & $n u p C$ & $\begin{array}{l}\text { Nucleoside transport } \\
\text { system protein }\end{array}$ & 80 & $85 / 85$ & E. coli $\mathrm{Sw}$ : P33031 \\
\hline
\end{tabular}


Table 1 (cont.)

\begin{tabular}{|c|c|c|c|c|c|c|c|}
\hline $\begin{array}{l}\text { Accession } \\
\text { no. }\end{array}$ & Fragment & Length (bp) & $\begin{array}{l}\text { Gene } \\
\text { homologues }\end{array}$ & $\begin{array}{l}\text { Putative } \\
\text { function* }\end{array}$ & $\begin{array}{c}\text { Identity } \\
(\%) \dagger\end{array}$ & $\begin{array}{l}\text { Amino } \\
\text { acid } \\
\text { overlap }\end{array}$ & $\begin{array}{c}\text { Organism/Accession } \\
\text { no. }\end{array}$ \\
\hline & & & $l y t R$ & $\begin{array}{l}\text { Putative negative } \\
\text { transcriptional } \\
\text { regulator for } \\
\text { lyt } A B C \text { major } \\
\text { autolysin operon }\end{array}$ & 38 & $105 / 118$ & B. subtilis $\mathrm{S}_{\mathrm{W}}$ : Q02115 \\
\hline \multirow[t]{2}{*}{ Y11140 } & bc48 & 1497 & or $B$ & $\begin{array}{l}\mathrm{U} \text { (similarity to } 2 \text { - } \\
\text { oxoacid ferredoxin } \\
\text { oxidoreductase) }\end{array}$ & 29 & $119 / 120$ & $\begin{array}{l}\text { Sulfolobus } \\
\text { acidocaldarius PIR: } \\
\text { JC4920 }\end{array}$ \\
\hline & & & $y m c B$ & $\begin{array}{l}\mathrm{U} \text { (similarity to } \\
y m c B)\end{array}$ & 75 & $212 / 226$ & B. subtilis $\mathrm{Em}$ : Z99112 \\
\hline \multirow[t]{3}{*}{ Y11171 } & bc542 & 2195 & $y o b N$ & $\begin{array}{l}\text { Putative L-amino } \\
\text { acid oxidase }\end{array}$ & 55 & $452 / 453$ & $\begin{array}{l}\text { B. subtilis Gb: } \\
\text { AF027868 }\end{array}$ \\
\hline & & & $b c 542 b$ & $\begin{array}{l}\mathrm{U} \text { (Similarity to } \\
y w c H \text { and } y t m \mathrm{O})\end{array}$ & 62 & $21 / 21$ & B. subtilis Sw : P39606 \\
\hline & & & & & 68 & $19 / 21$ & $\begin{array}{l}\text { B. subtilis Gb: } \\
\text { AF008220 }\end{array}$ \\
\hline \multirow[t]{2}{*}{ Y 10908} & bc75 & 1911 & cys $A$ & $\begin{array}{l}\text { Sulfate transport } \\
\text { ATP-binding } \\
\text { protein }\end{array}$ & 46 & $233 / 233$ & $\begin{array}{l}\text { Synechococcus PCC } \\
7942 \text { Sw: P14788 }\end{array}$ \\
\hline & & & wapA & $\begin{array}{l}\text { Wall-associated } \\
\text { protein }\end{array}$ & 40 & $197 / 231$ & $\begin{array}{l}\text { B. stearothermophilus } \\
\text { Sw: P42018 }\end{array}$ \\
\hline \multirow[t]{5}{*}{ Y09323 } & bc332 & 1606 & $b c 332 a$ & $\begin{array}{l}\text { U (similarity to } \\
\text { purine nucleoside } \\
\text { phosphorylase } \\
\text { homologue } p f s \text { ) }\end{array}$ & 26 & $204 / 216$ & E. coli $\mathrm{Sw}_{\mathrm{w}} \mathrm{P} 24247$ \\
\hline & & & $b c 332 b$ & $\begin{array}{l}\mathrm{U} \text { (similarity to } S \text {. } \\
\text { griseus pbnO }\end{array}$ & 30 & $110 / 145$ & $\begin{array}{l}\text { Streptomyces griseus } \\
\text { DDBJ: D29661 }\end{array}$ \\
\hline & & & & $\begin{array}{l}\text { regulatory protein } \\
\text { for C-P lyase and to } \\
\text { B. subtilis ywnH) }\end{array}$ & 28 & $138 / 145$ & B. subtilis Em: Z99122 \\
\hline & & & $I S 231$ & $\begin{array}{l}\text { Similarity }(90 \% \text { in } \\
67 / 70 \text { bp) to } \\
\text { insertion sequence } \\
\text { IS } 231 \mathrm{G} \text { (embl: } \\
\text { BACINSAA) }\end{array}$ & & & \\
\hline & & & gerPF & $\begin{array}{l}\text { Probable spore- } \\
\text { germination protein } \\
\mathrm{F}\end{array}$ & 89 & $39 / 39$ & $\begin{array}{l}\text { B. cereus } \mathrm{Gb} \text { : } \\
\text { AF053927 }\end{array}$ \\
\hline Y11218 & bc521 & 1796 & $s p 5 k \_2$ & $\begin{array}{l}\text { Similarity to stage } \mathrm{V} \\
\text { sporulation protein } \\
\mathrm{K}\end{array}$ & 41 & $280 / 598$ & B. subtilis $\mathrm{Sw}$ : $\mathrm{P} 27643$ \\
\hline Y11172 & bc 543 & 90 & $a p p C$ & $\begin{array}{l}\text { Oligopeptide } \mathrm{ABC} \\
\text { transport system } \\
\text { permease protein }\end{array}$ & 55 & $29 / 29$ & B. subtilis $\mathrm{Sw}$ : $\mathrm{P} 42063$ \\
\hline Y09255 & bc86 & 245 & $d n a I$ & Primosomal protein & 64 & $73 / 74$ & B. subtilis $\mathrm{Sw}: \mathrm{P} 06567$ \\
\hline \multirow[t]{2}{*}{ Y09719 } & bc69 & 2475 & mem1 & $\begin{array}{l}\mathrm{U} \text { (similar to } \\
\text { transmembrane } \\
\text { proteins) }\end{array}$ & 33 & $193 / 196$ & $\begin{array}{l}\text { Saccharomyces } \\
\text { cerevisiae PIR: } \\
\text { S69588 }\end{array}$ \\
\hline & & & $b c t L$ & $\begin{array}{l}\text { Membrane transport } \\
\text { protein, major } \\
\text { facilitator } \\
\text { superfamily } \\
\text { (Økstad } \text { et al., } \\
1997)\end{array}$ & 29 & $401 / 441$ & B. subtilis $\mathrm{Sw}$ : $\mathrm{P} 23054$ \\
\hline
\end{tabular}


Table 1 (cont.)

\begin{tabular}{|c|c|c|c|c|c|c|c|}
\hline $\begin{array}{l}\text { Accession } \\
\text { no. }\end{array}$ & Fragment & Length (bp) & $\begin{array}{l}\text { Gene } \\
\text { homologues }\end{array}$ & $\begin{array}{l}\text { Putative } \\
\text { function" }\end{array}$ & $\begin{array}{l}\text { Identity } \\
(\%) \dagger\end{array}$ & $\begin{array}{l}\text { Amino } \\
\text { acid } \\
\text { overlap }\end{array}$ & $\begin{array}{c}\text { Organism/Accession } \\
\text { no. }\end{array}$ \\
\hline & & & $g l r$ & Glutamate racemase & 59 & $267 / 269$ & B. subtilis Sw : P94556 \\
\hline \multirow[t]{2}{*}{ X98455 } & bc203 & 5142 & $y c b B$ & $\begin{array}{l}\text { Putative two- } \\
\text { component system } \\
\text { response regulator } \\
\text { (Lindback et al., } \\
\text { 1997) }\end{array}$ & 52 & $306 / 310$ & B. subtilis Sw: P40759 \\
\hline & & & $s n f 2$ & $\begin{array}{l}\text { Putative SNF2 } \\
\text { helicase (Lindbäck } \\
\text { et al., 1997) }\end{array}$ & 38 & $506 / 1066$ & $\begin{array}{l}\text { Synechocystis sp. } \\
\text { DDBJ : D90916 }\end{array}$ \\
\hline \multirow[t]{3}{*}{ A J010131 } & bc333 & 2303 & $y k v W$ & $\begin{array}{l}\text { Putative heavy- } \\
\text { metal-transporting } \\
\text { ATPase }\end{array}$ & 60 & $222 / 225$ & B. subtilis Em: Z99111 \\
\hline & & & $y f k H$ & $\begin{array}{l}\mathrm{U} \text { (similarity to } \\
y f k H)\end{array}$ & 51 & $265 / 289$ & B. subtilis Em: Z99108 \\
\hline & & & $b c 333 c$ & $\mathrm{U}$ & & & \\
\hline \multirow[t]{4}{*}{ AJ010111 } & bc9 & 6169 & $p y c$ & Pyruvate carboxylase & 76 & $982 / 984$ & $\begin{array}{l}\text { B. stearothermophilus } \\
\text { DDBJ : D83706 }\end{array}$ \\
\hline & & & $\operatorname{ctaA}$ & $\begin{array}{l}\text { Cytochrome } a a_{3} \\
\text { controlling protein }\end{array}$ & 57 & $307 / 345$ & $\begin{array}{l}\text { B. stearothermophilus } \\
\text { DDBJ : D70843 }\end{array}$ \\
\hline & & & $c t a B$ & $\begin{array}{l}\text { Cytochrome } c \\
\text { oxidase assembly } \\
\text { factor }\end{array}$ & 59 & $300 / 307$ & B. subtilis $\mathrm{S}_{\mathrm{w}}$ : $\mathrm{P} 24009$ \\
\hline & & & $c t a C$ & $\begin{array}{l}\text { Cytochrome } c \\
\text { oxidase subunit II }\end{array}$ & 53 & $223 / 227$ & $\begin{array}{l}\text { B. stearothermophilus } \\
\text { DDBJ: D70843 }\end{array}$ \\
\hline AJ010134 & bc8 & 408 & $b c 8 a$ & $\begin{array}{l}\mathrm{U} \text { (similarity to nasR } \\
\text { nitrate regulatory } \\
\text { protein) }\end{array}$ & 31 & $63 / 130$ & $\begin{array}{l}\text { Klebsiella pneumoniae } \\
\text { PIR: A55859 }\end{array}$ \\
\hline \multirow[t]{2}{*}{ AJ010135 } & bc121 & 380 & $r p s B$ & $\begin{array}{l}\text { Ribosomal protein } \\
\text { S2 }\end{array}$ & 98 & $48 / 51$ & B. subtilis Em: Z99112 \\
\hline & & & $t s f$ & Elongation factor $\mathrm{Ts}$ & 96 & $24 / 24$ & B. subtilis $\mathrm{Em}$ : Z99112 \\
\hline A J010136 & bc122 & 314 & $y t i P$ & $\mathrm{U}$ (similarity to $y t i P)$ & 66 & $102 / 104$ & $\begin{array}{l}\text { B. subtilis } \mathrm{Gb} \text { : } \\
\text { AF00822 }\end{array}$ \\
\hline AJ010331 & bc16 & 252 & bla1 & $\beta$-Lactamase I & 88 & $60 / 68$ & B. cereus PIR : S03167 \\
\hline AJ010132 & bc891 & 498 & $b c 89 a$ & $\begin{array}{l}\text { U (similarity to } \\
\text { inosine-uridine } \\
\text { preferring } \\
\text { nucleoside } \\
\text { hydrolase/purine } \\
\text { nucleosidase) }\end{array}$ & 32 & $148 / 166$ & $\begin{array}{l}\text { Crithidia fasciculata } \\
\text { Sw: Q27546 }\end{array}$ \\
\hline A J010133 & bc892 & 452 & $y v g N$ & $\begin{array}{l}\text { Putative } \\
\text { dehydrogenase }\end{array}$ & 78 & $127 / 130$ & B. subtilis $\mathrm{Em}$ : Z99121 \\
\hline A J010112 & bc85 & 125 & gerPF & $\begin{array}{l}\text { Probable spore } \\
\text { germination protein } \\
\text { F }\end{array}$ & 100 & $33 / 33$ & $\begin{array}{l}\text { B. cereus } \mathrm{Gb} \text { : } \\
\text { AF } 053927\end{array}$ \\
\hline A J010129 & bc421 & 615 & $y x a A$ & $\begin{array}{l}\mathrm{U} \text { (similarity to } \\
y x a A)\end{array}$ & 48 & $64 / 69$ & B. subtilis Sw: P42100 \\
\hline A J010130 & bc422 & 74 & $b d h$ & $\begin{array}{l}\text { Putative homoserine } \\
\text { dehydrogenase }\end{array}$ & 60 & $23 / 24$ & $\begin{array}{c}\text { Synechocystis PCC } \\
6803 \text { Sw : P52986 }\end{array}$ \\
\hline AJ010137 & bc2 2 & 107 & $y d h E$ & $\begin{array}{l}\text { Putative glycosyl } \\
\text { transferase }\end{array}$ & 51 & $29 / 35$ & B. subtilis Em: Z99107 \\
\hline Aj010138 & bc201 & 3252 & spoIIQ & $\begin{array}{l}\text { Stage II sporulation; } \\
\text { required for } \\
\text { completion of } \\
\text { engulfment }\end{array}$ & 45 & $152 / 196$ & B. subtilis Em: Z99122 \\
\hline
\end{tabular}


Table 1 (cont.)

\begin{tabular}{|c|c|c|c|c|c|c|c|}
\hline $\begin{array}{l}\text { Accession } \\
\text { no. }\end{array}$ & Fragment & Length (bp) & $\begin{array}{c}\text { Gene } \\
\text { homologues }\end{array}$ & $\begin{array}{l}\text { Putative } \\
\text { function* }\end{array}$ & $\begin{array}{c}\text { Identity } \\
(\%) \dagger\end{array}$ & $\begin{array}{l}\text { Amino } \\
\text { acid } \\
\text { overlap }\end{array}$ & $\begin{array}{c}\text { Organism/Accession } \\
\text { no. }\end{array}$ \\
\hline & & & spollid & $\begin{array}{l}\text { Stage III sporulation } \\
\text { protein }\end{array}$ & 98 & $90 / 90$ & $\begin{array}{l}\text { B. thuringiensis DDBJ: } \\
\text { D28169 }\end{array}$ \\
\hline & & & $m b l$ & $\begin{array}{l}\text { Cell shape- } \\
\text { determination-like } \\
\text { protein (mreB-like) }\end{array}$ & 87 & $332 / 334$ & B. subtilis $\mathrm{Sw}_{\mathrm{w}} \mathrm{P} 39751$ \\
\hline & & & $b c r 1$ & $\begin{array}{l}\text { Bacillus cereus repeat } \\
1 ; 155 \mathrm{nt} \text { sequence } \\
\text { repeated in the } B . \\
\text { cereus genome }\end{array}$ & & & \\
\hline & & & $f a b Z$ & $\begin{array}{l}\text { Putative } \\
\text { hydroxymyristoyl- } \\
\text { (acyl carrier } \\
\text { protein) } \\
\text { dehydratase }\end{array}$ & 79 & $42 / 42$ & B. subtilis $\mathrm{Em}$ : Z83337 \\
\hline \multirow[t]{3}{*}{ AJ010139 } & bc295 & 2866 & fumA & $\begin{array}{l}\text { Class I Fe-S } \\
\text { fumarase }\end{array}$ & 77 & $269 / 269$ & $\begin{array}{l}\text { B. stearothermophilus } \\
\text { Sw: Q04718 }\end{array}$ \\
\hline & & & $y f j S$ & $\begin{array}{l}\text { Polysaccharide } \\
\text { deacetylase }\end{array}$ & 57 & $255 / 260$ & $\begin{array}{l}\text { B. stearothermophilus } \\
\text { Sw: Q04729 }\end{array}$ \\
\hline & & & alkE & $\begin{array}{l}\text { DNA-3- } \\
\text { methyladenine } \\
\text { glycosidase II }\end{array}$ & 45 & $287 / 287$ & B. subtilis $\mathrm{Em}$ : Z99108 \\
\hline AJ010140 & bc296 & 1356 & alkC & $\begin{array}{l}\text { DNA alkylation } \\
\text { repair enzyme }\end{array}$ & 40 & $243 / 256$ & B. subtilis Em: Y14080 \\
\hline \multirow[t]{3}{*}{ A J010128 } & bc297 & 2683 & $b c 297 a$ & $\begin{array}{l}\mathrm{U} \text { (similarity to } \\
y v b W \text { ) }\end{array}$ & 23 & $196 / 203$ & $\begin{array}{l}\text { B. subtilis SubtiList: } \\
\text { yvbW }\end{array}$ \\
\hline & & & alkD & $\begin{array}{l}\text { DNA alkylation } \\
\text { repair enzyme; } \\
\text { shown by } \\
\text { complementation, } \\
\text { I. Alseth and others, } \\
\text { unpublished results }\end{array}$ & & & \\
\hline & & & $g l y S$ & $\begin{array}{l}\text { Glycyl-tRNA } \\
\text { synthetase }\end{array}$ & 50 & $214 / 217$ & $\begin{array}{l}\text { M. tuberculosis } \mathrm{Em}: \\
\text { Z95208 }\end{array}$ \\
\hline \multirow[t]{6}{*}{ AJ000394 } & bc298 & 4392 & celR & $\begin{array}{l}\text { Putative } \\
\text { transcriptional } \\
\text { regulator of } \\
\text { cellobiose } \\
\text { phosphotransferase } \\
\text { system operon }\end{array}$ & 38 & $460 / 482$ & $\begin{array}{l}\text { B. stearothermophilus } \\
\text { PIR: A49898 }\end{array}$ \\
\hline & & & $b c 298 b$ & $\begin{array}{l}\mathrm{U} \text { (similarity to } \\
\text { zebrafish chordin) }\end{array}$ & 30 & $126 / 137$ & $\begin{array}{l}\text { Danio rerio } \mathrm{Gb} \text { : } \\
\text { AF034606 }\end{array}$ \\
\hline & & & $c b r A$ & $\begin{array}{l}\text { Membrane transport } \\
\text { protein. Similarity } \\
\text { to chromate } \\
\text { resistance proteins }\end{array}$ & 25 & $379 / 393$ & $\begin{array}{l}\text { Synechocys. PCC } 6803 \\
\text { DDBJ : D } 90916\end{array}$ \\
\hline & & & & $\begin{array}{l}\text { Cellobiose } \\
\text { phosphotransferase }\end{array}$ & 50 & $230 / 234$ & $\begin{array}{l}\text { B. stearothermophilus } \\
\text { PIR: D49898 }\end{array}$ \\
\hline & & & $b c r 1$ & $\begin{array}{l}\text { B. cereus repeat } 1 \text {; } \\
15.5 \mathrm{nt} \text { sequence } \\
\text { repeated in the } B . \\
\text { cereus genome }\end{array}$ & & & \\
\hline & & & celF & $\begin{array}{l}\text { Putative phospho- } \beta \text { - } \\
\text { glucosidase }\end{array}$ & 78 & $9 / 9$ & $\begin{array}{l}\text { B. subtilis SubtiList: } \\
\text { licH }\end{array}$ \\
\hline
\end{tabular}


Table 1 (cont.)

\begin{tabular}{|c|c|c|c|c|c|c|c|}
\hline $\begin{array}{l}\text { Accession } \\
\text { no. }\end{array}$ & Fragment & Length (bp) & $\begin{array}{l}\text { Gene } \\
\text { homologues }\end{array}$ & $\begin{array}{l}\text { Putative } \\
\text { function* }\end{array}$ & $\begin{array}{c}\text { Identity } \\
(\%) \dagger\end{array}$ & $\begin{array}{l}\text { Amino } \\
\text { acid } \\
\text { overlap }\end{array}$ & $\begin{array}{c}\text { Organism/Accession } \\
\text { no. }\end{array}$ \\
\hline \multirow{7}{*}{ A J007788 } & \multirow{7}{*}{ bc299 } & \multirow{7}{*}{6006} & & & 100 & $9 / 9$ & $\begin{array}{l}\text { B. cereus } \mathrm{Em} \text { : } \\
\text { A J007952 }\end{array}$ \\
\hline & & & $i l v D$ & $\begin{array}{l}\text { Isoleucine } \\
\text { biosynthesis } \\
\text { enzyme; dihydroxy- } \\
\text { acid dehydratase }\end{array}$ & 60 & $355 / 357$ & B. subtilis Sw: P05791 \\
\hline & & & $i l v A$ & $\begin{array}{l}\text { Isoleucine } \\
\text { biosynthesis } \\
\text { enzyme; threonine } \\
\text { deaminase }\end{array}$ & 64 & $417 / 420$ & B. subtilis Sw: P37946 \\
\hline & & & $\operatorname{cap} A$ & $\begin{array}{l}\mathrm{U} \text { (similarity to } B \text {. } \\
\text { anthracis CapA }\end{array}$ & 29 & $307 / 368$ & $\begin{array}{l}\text { B. anthracis Sw: } \\
\text { P19579 }\end{array}$ \\
\hline & & & & $\begin{array}{l}\text { protein required for } \\
\text { biosynthesis of type } \\
\text { I capsular } \\
\text { polysaccharide) }\end{array}$ & 29 & $337 / 368$ & B. subtilis Em: Z92954 \\
\hline & & & $b c 299 d$ & $\mathrm{U}$ & & & \\
\hline & & & $b c 299 e$ & $\mathrm{U}$ & & & \\
\hline \multirow[t]{6}{*}{ A J007510 } & \multirow[t]{6}{*}{ bc210 } & \multirow[t]{6}{*}{10915} & $m c r B$ & $\begin{array}{l}\text { Putative DNA } \\
\text { restriction enzyme }\end{array}$ & 35 & $281 / 343$ & $\begin{array}{l}\text { M. thermoauto- } \\
\text { trophicum Gb: } \\
\text { AE000833 }\end{array}$ \\
\hline & & & $b c 210 b$ & $\begin{array}{l}\mathrm{U} \text { (similarity to SNF2 } \\
\text { helicase) }\end{array}$ & 20 & $200 / 257$ & $\begin{array}{l}\text { Plasmodium } \\
\text { falciparum } \mathrm{Gb}: \\
\text { AF003086 }\end{array}$ \\
\hline & & & $t 3 \bmod$ & $\begin{array}{l}\text { Type III restriction- } \\
\text { modification system } \\
\text { Mod protein }\end{array}$ & 50 & $677 / 690$ & $\begin{array}{l}\text { S. typhimurium } \mathrm{Sw}_{\mathrm{w}} \\
\text { P40814 }\end{array}$ \\
\hline & & & t3res & $\begin{array}{l}\text { Type III restriction- } \\
\text { modification system } \\
\text { Res protein (Hegna } \\
\text { et al., 1992) }\end{array}$ & 63 & $946 / 988$ & $\begin{array}{l}\text { S. typhimurium Sw: } \\
\text { P40815 }\end{array}$ \\
\hline & & & $b c 210 e$ & $\begin{array}{l}\mathrm{U} \text { (similarity to } \\
\text { Borrelia burgdorferi } \\
\text { putative coding } \\
\text { region } \mathrm{BB} 0553 \text { ) }\end{array}$ & 23 & $222 / 431$ & $\begin{array}{l}\text { Bor. burgdorferi Gb: } \\
\text { AE } 001157\end{array}$ \\
\hline & & & $b c 210 f$ & $\begin{array}{l}\mathrm{U} \text { (similarity to SNF2 } \\
\text { helicases) }\end{array}$ & 23 & $472 / 631$ & B. cereus Em: X98455 \\
\hline
\end{tabular}

${ }^{*} \mathrm{U}$, unknown function (weak or no similarity to database entries, or similarity to proteins of unknown function).

† ORFs were used to search a non-redundant set of database entries from SWISS-PROT, GenPept (translated GenBank), PDB, SPUpdate and PIR using the BLAST search algorithm at the National Center for Biotechnology Information (NCBI), Bethesda, MD, USA (http://www.ncbi.nim.nih.gov).

biodegradable than other less substituted s-triazine ring compounds (University of Minnesota Biocatalysis/ Biodegradation Database: http://www.labmed.umn. edu/umbbd/atr/atr_map.html). The presence in $B$. cereus of a $\operatorname{trz} A$ gene, encoding an $\mathrm{N}$-ethylammeline chlorohydrolase homologue novel to bacilli, could allow $B$. cereus to degrade herbicides in its natural soil habitat. The role of the or $B$ gene encoding a protein similar to the $\beta$-chain of 2-oxoacid: ferredoxin oxidoreductase, a key enzyme of the archaeal tricarboxylic acid cycle (Iwasaki et al., 1995), is unclear. Putative orB homo- logues have been found in the archaea Sulfolobus acidocaldarius and Methanobacterium thermoautotrophicum, but have only been identified in one other eubacterium, the thermophile Thermus thermophilus.

\section{B. subtilis gene organization is not conserved in $B$. cereus}

Using previous allocations of the anonymous probes to the physical map (Carlson et al., 1992; Carlson \& 
(a)

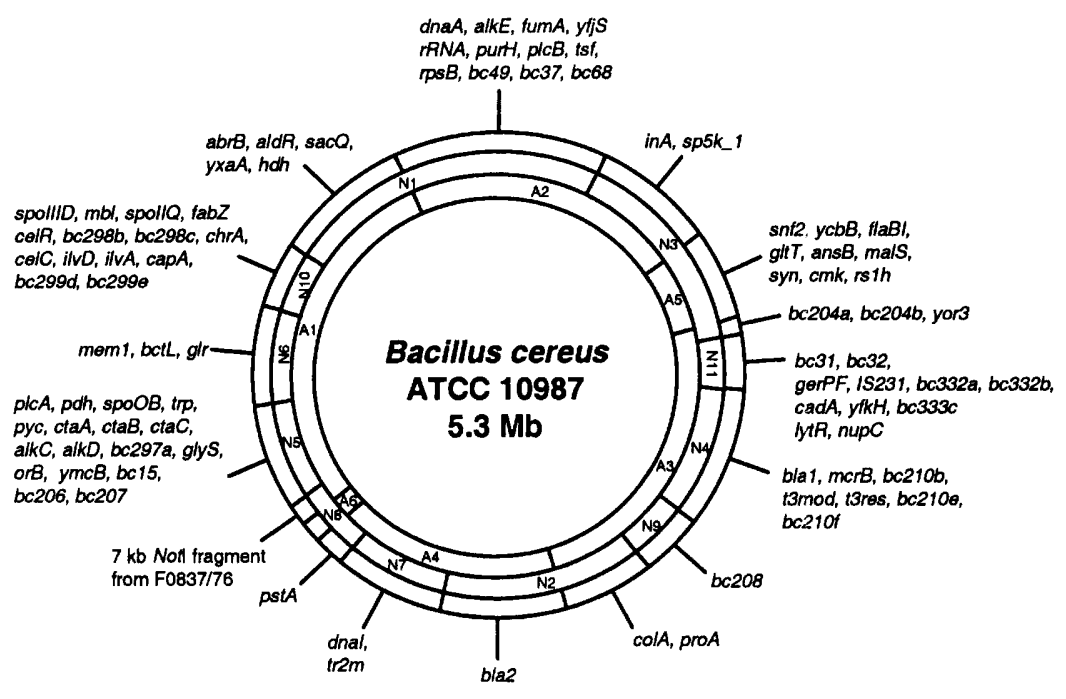

(b)

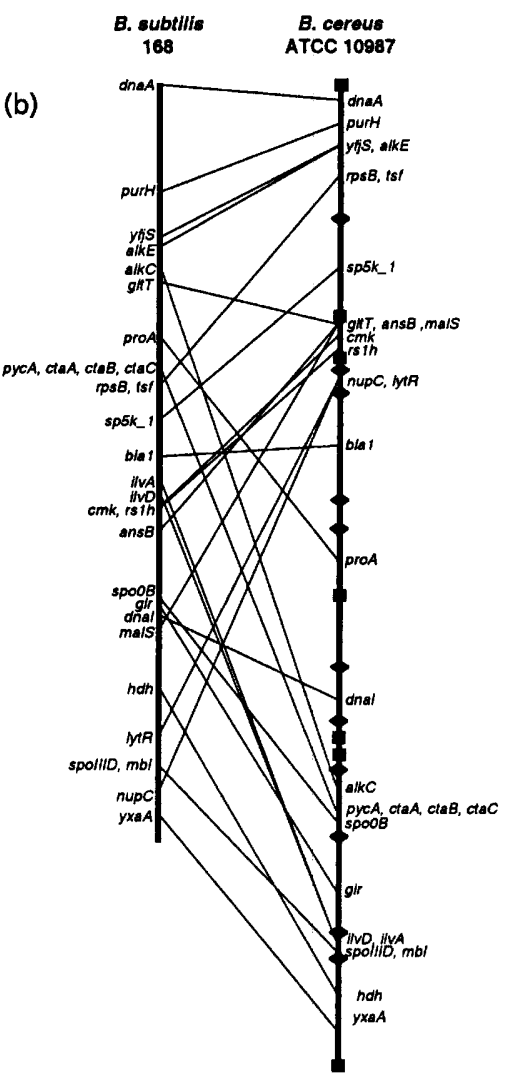

Fig. 1. (a) Physical map of the B. cereus ATCC 10987 chromosome. Inner circle, Ascl fragments; middle circle, Notl fragments. Restriction fragments are ordered according to size, with the lowest number corresponding to the largest fragment. (b) Comparison of gene localization on the B. cereus ATCC 10987 and B. subtilis 168 chromosomes. Notl sites and $A s c l$ sites are indicated by diamonds and squares, respectively. Both chromosomes are shown linearized, B. subtilis with $d n a A$ as the first gene on top, $B$. cereus with the region common to N1 and A1 restriction fragments on top. The comparison was drawn to obtain maximum similarity between the maps.

Kolstø, 1994), 64 of the identified genes/ORFs were assigned positions on the B. cereus ATCC 10987 chromosome (Fig. 1a). The updated map contains a total of 91 markers, of which 80 are genes/ORFs of known or unknown function, one is IS231, and 10 are anonymous. To examine whether the global gene order was conserved between $B$. cereus and $B$. subtilis, we compared the chromosomal location of 30 putative orthologues using the $B$. cereus ATCC 10987 physical map and the $B$. subtilis 168 genome sequence (Kunst et al., 1997). The orthologues did not exhibit any apparent conservation of organization (Fig. 1b), but were, by contrast, arranged in an apparently random manner. This is in agreement with what has been observed when comparing the genomes of the distantly related bacteria Haemophilus influenzae, Mycoplasma genitalium and Methanococcus jannaschii (Kolstø, 1997), and may indicate that gene organization is generally not conserved even between members of the same genus, like $B$. subtilis and $B$. cereus. Similar results were found when comparing the position of nine equivalent genes in alkaliphilic Bacillus firmus OF4 with B. subtilis 168 (Grønstad et al., 1998).
There were, however, examples where genes exhibited an identical clustering in $B$. cereus and $B$. subtilis: (1) spoIIID and $m b l$; (2) pycA, ctaA, ctaB, and ctaC; (3) $r p s B$ and $t s f$. It is, however, interesting to note that although the local gene order was conserved, the chromosomal location of the three loci was very different in the two bacteria (Fig. 1b). Furthermore, the $r p s B-t s f$ gene order encoding ribosomal protein $\mathrm{S} 2$ and elongation factor Ts is one of the very few examples of gene pairs for which orthologues are close to being universally clustered in eubacteria, being conserved in 10 out of 14 species where both genes have been sequenced. Additionally, putative homologues of two other B. cereus gene pairs were located in the vicinity of each other in $B$. subtilis, although lacking a tight physical coupling. (1) The branched chain amino acid synthesis genes $i l v D$ and ilvA were in B. subtilis 168 separated by an $8.4 \mathrm{~kb}$ region harbouring nine genes. Similarly, (2) the putative polysaccharide deacetylase gene $y f j S$ and the DNA-3-methyladenine glycosidase II gene alkE were $2.8 \mathrm{~kb}$ apart in $B$. subtilis, separated by two ORFs with similarity to 3hydroxyisobutyrate dehydrogenase $(y f j R)$ and a divalent cation transporter $(y f j Q)$, respectively. 
(a)

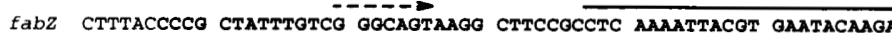

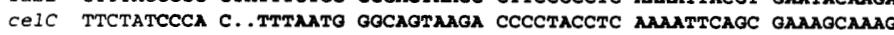

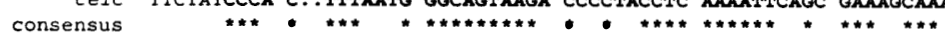

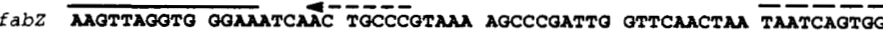

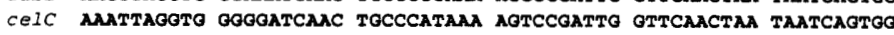

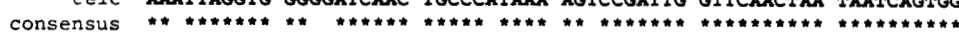

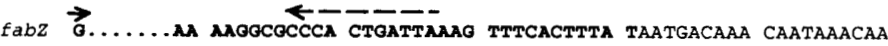

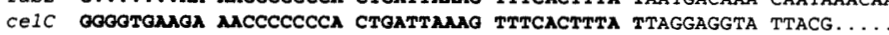

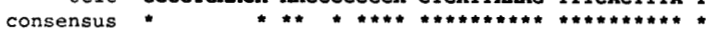

(b)

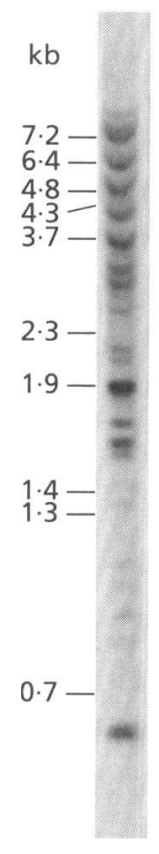

(c)

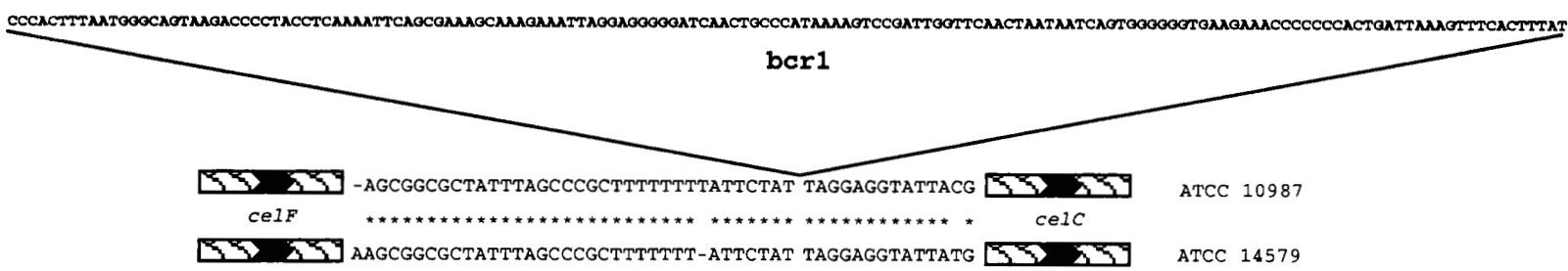

Fig. 2. (a) Two gene loci from B. cereus ATCC 10987 and ATCC 14579 containing the 154 bp bcr1 repeat, with the defined bcr1 sequence in bold. Inverted repeats within bcr1 are marked by dashed arrows. The region covered by the oligonucleotide probe is indicated by the solid line above the sequences. (b) Analysis of $B$. cereus ATCC 10987 genomic DNA using $37 \mathrm{nt}$ of the bcr1 repeat as probe. Genomic DNA was digested with Hincll, electrophoresed and blotted to a nylon membrane. Numbers indicate the positions of the molecular size marker ( $\lambda$ DNA digested with BstEII, New England Biolabs). (c) Comparison of the celF-celC gene locus from B. cereus strains ATCC 10987 and ATCC 14579. Apart from the bcr1 insertion, the intergenic regions were conserved in the two strains.

\section{A novel repeat sequence in the $B$. cereus genome}

Sequencing of the celR-bc298a-chrA-celC-celF gene locus showed that an intergenic 155 bp sequence in front of the celC gene was highly similar to a sequence upstream of fabZ in the spoIIQ-spollID-mbl-fabZ gene locus (Fig. 2a). We have named this novel repeated sequence $b c r 1$ (Bacillus cereus repeat 1 ). Repeats of $b c r 1$ were also found upstream of the $h b l$ enterotoxin locus from strain ATCC 14579 (O. A. Økstad \& A.-B. Kolstø, unpublished results), and upstream of bctL (Økstad et al., 1997) and the bct504a gene from the same strain (data not shown). The $b c r 1$ sequences exhibited 78-93\% pairwise identity, and a multiple alignment of the repeats showed that $63 \%$ of the nucleotide positions were conserved in all five loci. The sequences displayed an inverted repeat character, and were in all instances found in non-coding regions.

To investigate the degree of $b c r 1$ repetition, an oligo- nucleotide probe of $37 \mathrm{bp}$ within $b c r 1$ was prepared, and blots of HincII-digested genomic DNA were hybridized with the probe. In strain ATCC 10987, 11 fragments hybridized strongly to the probe, while 11 fragments displayed weaker hybridization intensity (Fig. 2b). Similar hybridization results were obtained with 16 other $B$. cereus and B. thuringiensis strains tested, indicating that $b c r 1$ may be ubiquitous in $B$. cereus and $B$. thuringiensis strains. The locations of the repeat within the genome may vary, as shown by the presence of $b c r 1$ in the $c e l C-c e l F$ locus in strain ATCC 10987, and its absence in the corresponding locus from strain ATCC 14579 (Fig. 2c). No hybridization by the $b c r 1$ probe to $B$. subtilis DNA was observed, and the sequence was not present when searching SubtiList or any other database. The $b c r 1$ repeat did not display similarity to any integron family sequences, including the repetitive sequence elements recently reported in Vibrio cholerae (Clark et al., 1997). 


\section{Concluding remarks}

E. coli and S. typhimurium, species thought to have differentiated 120-160 million years ago (Ochman \& Wilson, 1987), have highly similar genetic maps. This led to the prediction that bacterial genomes would turn out to be generally stable, exhibiting a conserved chromosomal gene organization. Subsequent studies have indicated that this is generally not the case. Bacteria may be divided into two subgroups in terms of genome stability, where one group consists of stable species whereas members of the other subgroup display a higher level of heterogeneity within the species (Fonstein \& Haselkorn, 1995). B. cereus clearly belongs to the latter group, with different strains showing a highly variable gene organization, particularly in certain regions of the genome (Carlson \& Kolstø, 1994). Salmonella typhi (Liu \& Sanderson, 1996), Pseudomonas stutzeri (Ginard et al., 1997) and Brucella suis (Jumas-Bilak et al., 1998) also belong to the high variability group, and homologous recombination between the $r r n$ loci seems to be the major cause of their heterogeneity. It is interesting to note that the organization of the $r r n$ gene operons within the clostridial chromosomes varies considerably (Cornillot et al., 1997). In fact, the rrn operon organization of Clostridium acetobutylicum was more similar to that of B. subtilis than to other clostridia. By contrast, B. subtilis 168 has, by the examination of a number of derived strains, been found to have a generally stable chromosome structure in agreement with the high genetic stability of the species (Itaya, 1993).

We have previously suggested that $B$. cereus strains have been subject to a high frequency of genome rearrangements (Carlson et al., 1992; Carlson \& Kolstø, 1993, 1994). The present study shows that the chromosomal gene organization in B. cereus ATCC 10987 is widely different from $B$. subtilis 168 . These results add to a growing number of studies indicating that bacteria exhibiting a conserved chromosomal gene organization may in fact be exceptions rather than the rule. We do not know if the $b c r 1$ sequence repeats may contribute to the chromosome variation and flexibility observed in strains of B. cereus and B. thuringiensis.

\section{ACKNOWLEDGEMENTS}

The authors are thankful to Ewa Jaroszewicz, Henning A. Johansen and Liv A. Bjørland for skilful technical assistance. The work was supported by grants from The Norwegian Research Council to A.B.K.

\section{REFERENCES}

Altschul, S. F., Gish, W., Miller, W., Myers, E. W. \& Lipman, D. J. (1990). Basic local alignment search tool. J Mol Biol 215, 403-410.

Altschul, S. F., Madden, T. L., Schäffer, A. A., Zhang, J., Zhang, Z., Miller, W. \& Lipman, D. J. (1997). Gapped BLAST and PSI-BLAST : a new generation of protein database search programs. Nucleic Acids Res 25, 3389-3402.

Aronson, A. I. (1993). The two faces of Bacillus thuringiensis: insecticidal proteins and post-exponential survival. Mol Microbiol 7, 489-496.
Carison, C. R. \& Kolstø, A.-B. (1993). A complete physical map of a Bacillus thuringiensis chromosome. J Bacteriol 175, 1053-1060.

Carlson, C. R. \& Kolstø, A.-B. (1994). A small (2.5 Mb) Bacillus cereus chromosome corresponds to a conserved region of a larger $(5 \cdot 3 \mathrm{Mb})$ Bacillus cereus chromosome. Mol Microbiol 13, 161-169.

Carlson, C. R., Grønstad, A. \& Kolstø, A.-B. (1992). Physical maps of the genomes of three Bacillus cereus strains. J Bacteriol 174, 3750-3756.

Carlson, C. R., Johansen, T. \& Kolstø, A.-B. (1996a). The chromosome map of Bacillus thuringiensis subsp. canadensis HD224 is highly similar to that of the Bacillus cereus type strain ATCC 14579. FEMS Microbiol Lett 141, 163-167.

Carlson, C. R., Johansen, T., Lecadet, M.-M. \& Kolstø, A.-B. (1996b). Genomic organization of the entomopathogenic bacterium Bacillus thuringiensis subsp. berliner 1715. Microbiology 142, 1625-1634.

Clark, C. A., Purins, L., Kaewrakon, P. \& Manning, P. A. (1997). VCR repetitive sequence elements in the Vibrio cholerae chromosome constitute a mega-integron. Mol Microbiol 26, 1137-1138.

Cornillot, E., Croux, C. \& Soucaille, P. (1997). Physical and genetic map of the Clostridium acetobutylicum ATCC 824 chromosome. $J$ Bacteriol 179, 7426-7434.

Devereux, J., Haeberli, P. \& Smithies, O. (1984). A comprehensive set of sequence analysis programs for the VAX. Nucleic Acids Res 12, 387-395.

Drobniewski, F. A. (1993). Bacillus cereus and related species. Clin Microbiol Rev 6, 324-338.

Feng, D. F. \& Doolittle, R. F. (1987). Progressive sequence alignment as a prerequisite to correct phylogenetic trees. $J \mathrm{Mol}$ Evol 25, 351-360.

Fonstein, M. \& Haselkorn, R. (1995). Physical mapping of bacterial genomes. J Bacteriol 177, 3361-3369.

Ginard, M., Lalucat, J., Tummler, B. \& Romling, U. (1997). Genome organisation of Pseudomonas stutzeri and resulting taxonomic and evolutionary considerations. Int J Syst Bacteriol 47, 132-143.

Gordon, R. E., Haynes, W. C. \& Pang C. H.-N. (1973). The Genus Bacillus. Agriculture Handbook no. 427. Washington, DC: United States Department of Agriculture.

Green, L. S., Laudenbach, D. E. \& Grossman, A. R. (1989). A region of a cyanobacterial genome required for sulfate transport. Proc Natl Acad Sci USA 86, 1949-1953.

Grønstad, A., Jaroszewicz, E., Ito, M., Sturr, M. G., Krulwich, T. A. \& Kolsto, A.-B. (1998). Physical map of alkaliphilic Bacillus firmus OF4 and detection of a large endogenous plasmid. Extremophiles 2, 447-453.

Hegna, I. K., Karlstrom, E. S., Lopez, R., Kristensen, T. \& Kolsto, A.-B. (1992). A type-III DNA restriction and modification system in Bacillus cereus. Gene 114, 149-150.

Higgins, D. G. \& Sharp, P. M. (1989). Fast and sensitive multiple sequence alignments on a microcomputer. Comput Appl Biosci 5, 151-153.

Himmelreich, R., Plagens, H., Hilbert, H., Reiner, B. \& Herrmann, R. (1997). Comparative analysis of the genomes of the bacteria Mycoplasma pneumoniae and Mycoplasma genitalium. Nucleic Acids Res 25, 701-712.

Itaya, M. (1993). Stability and asymmetric replication of the Bacillus subtilis 168 chromosome structure. J Bacteriol 175, 741-749.

Iwasaki, T., Wakagi, T. \& Oshima, T. (1995). Ferredoxin-dependent redox system of a thermoacidophilic archaeon, Sulfolobus sp. 
strain 7. Purification and characterization of a novel reduced ferredoxin-reoxidizing iron-sulfur flavoprotein. J Biol Chem 270, 17878-17883.

Jumas-Bilak, E., Michaux-Charachon, S., Bourg, G., O'Callaghan, D. \& Ramuz, M. (1998). Differences in chromosome number and genome rearrangements in the genus Brucella. Mol Microbiol 27, 99-106.

Kolstø, A.-B. (1997). Dynamic bacterial genome organisation. Mol Microbiol 24, 241-248.

Kolstø, A.-B., Grønstad, A. \& Oppegaard, H. (1990). Physical map of the Bacillus cereus chromosome. J Bacteriol 172, 3821-3825.

Koonin, E. V., Mushegian, A. R. \& Rudd, K. E. (1996). Sequencing and analysis of bacterial genomes. Curr Biol 6, 404-416.

Kunst, F., Ogasawara, N., Moszer, I. \& 148 other authors (1997). The complete genome sequence of the Gram-positive bacterium Bacillus subtilis. Nature 390, 249-256.

Lindbäck, T., Kolstø, A.-B. \& Kolst, A. B. (1997). A Bacillus cereus member of the SNF2 family. Microbiology 143, 171-174.

Liu, S. L. \& Sanderson, K. E. (1996). Highly plastic chromosomal organization in Salmonella typhi. Proc Natl Acad Sci USA 93, 10303-10308.

Logan, D. T., Mazauric, M. H., Kern, D. \& Moras, D. (1995). Crystal structure of glycyl-tRNA synthetase from Thermus thermophilus. EMBO J 14, 4156-4167.

Lövgren, A., Carlson, C. R., Eskils, K. \& Kolstø, A.-B. (1998). Localization of putative virulence genes on a physical map of the Bacillus thuringiensis subsp. gelechiae chromosome. Curr Microbiol 37, 245-250.

Needleman, S. B. \& Wunsch, C. D. (1970). A general method applicable to the search for similarities in the amino acid sequence of two proteins. $J \mathrm{Mol} \mathrm{Biol} \mathrm{48,} \mathrm{443-453.}$

Ochman, H. \& Wilson, A. C. (1987). Evolution in bacteria : evidence for a universal substitution rate in cellular genomes. Mol Evol 26, 74-86.
Økstad, O. A., Grønstad, A., Lindbäck, T. \& Kolstø, A.-B. (1997). Insertional inactivation of a $\operatorname{Tet}(\mathrm{K}) / \operatorname{Tet}(\mathrm{L})$ like transporter does not eliminate tetracycline resistance in Bacillus cereus. FEMS Microbiol Lett 154, 181-186.

Pearson, W. R. \& Lipman, D. J. (1988). Improved tools for biological sequence comparison. Proc Natl Acad Sci USA 85, 2444-2448.

Priest, F. G. (1993). Systematics and ecology of Bacillus. In Bacillus subtilis and Other Gram-positive Bacteria: Biochemistry, Physiology, and Molecular Genetics, pp. 3-16. Edited by A. L. Sonenshein, J. A. Hoch \& R. Losick. Washington, DC: American Society for Microbiology.

Sambrook, J., Fritsch, E. F. \& Maniatis, T. (1989). Molecular Cloning: a Laboratory Manual, 2nd edn. Cold Spring Harbor, NY: Cold Spring Harbor Laboratory.

Sanger, F., Nicklen, S. \& Coulson, A. R. (1977). DNA-sequencing with chain-terminating inhibitors. Proc Natl Acad Sci USA 74, 5463-5467.

Smith, D. R., Doucette-Stamm, L. A., Deloughery, C. \& 34 other authors (1997). Complete genome sequence of Methanobacterium thermoautotrophicum deltaH: functional analysis and comparative genomics. J Bacteriol 179, 7135-7155.

Smith, N. R., Gordon, R. E. \& Clark, F. E. (1952). In Aerobic Sporeforming Bacteria, pp. 56-67. Washington, DC: US Department of Agriculture.

Tatusov, R. L., Mushegian, A. R., Bork, P., Brown, N. P., Hayes, W. S., Borodovsky, M., Rudd, K. E. \& Koonin, E. V. (1996). Metabolism and evolution of Haemophilus influenzae deduced from a whole-genome comparison with Escherichia coli. Curr Biol 6, 279-291.

Received 21 September 1998; revised 10 November 1998; accepted 18 November 1998. 